\title{
Unified Description of the Onboard Equipment Model on the Basis of the «Simulation Model Portability» Standard
}

\author{
Ludmila Nozhenkova, Olga Isaeva, Evgeniy Gruzenko, Andrey Koldyrev, Aleksey Markov, Artem Belorusov and \\ Rodion Vogorovskiy \\ Institute of Computational Modelling of the Siberian Branch of the Russian Academy of Sciences, Akademgorodok 50/44, \\ 660036 Krasnoyarsk, Russia
}

\begin{abstract}
The article presents a unified description of the onboard equipment model for building simulation modeling infrastructure based on the international «Simulation Model Portability" (SMP2) standard. The model contains structuralparametric and functional description of the elements determining its composition and features, as well as the method of control of transitions between the conditions under modeling. We suggest the principles of unification allowing to build a model in the terms of the subject area, with their following translation to the program elements of the model working in accordance with the SMP2 standard.
\end{abstract}

Keywords-simulation model portability; spacecraft; onboard equipment; command and measuring system

\section{INTRODUCTION}

The world space industry consists of manufacturers of space systems of different countries. Besides the historical leaders in this area - NASA (USA), ESA (Europe) and Roscosmos (Russia) there are comparatively young centers of rocket-and-space technologies: JAXA in Japan, CNSA in China, ISRO in India, etc. One of the important directions nowadays is integration of space projects and standardization of the technical production processes. In order to support the tasks of projecting and production of space equipment, scientists create simulation models to perform the required characteristics and properties of space systems. The models are created by different companies, each of them presenting its own highly specialized direction, being not able to create actual models of all interacting subsystems. It is necessary to integrate them into a one complex model allowing to make simulation tests with the ability for quick modification and expansion. The principles of accumulation and usage of the models are regulated by the SMP - Simulation Model Portability standard (SMP2, ECSS E-40-07 in the current edition), recommended by the European Cooperation for Space Standardization which sets the universal approaches to organization of the modeling systems for integration of separate models into complex solutions, their transferability between the simulation environments and operating systems [1]. The standard determines the rules of providing interoperability and transferability of simulation models between the heterogeneous modeling systems.

The software environments realizing the SMP standard are
Simulation Infrastructures. There exists a number of simulation modeling infrastructure designs. Some of the main of them are: SimTG infrastructure used in the Astrium Satellites, described by Claude Cazenave, William Arrouy [2], SimSAT structure is used by the European Space Agency, the authors are J. Eggleston, H. Boyer, D. van der Zee, etc [3]. This technology is used to build simulators of the mission control centers: SWARMSIM, authors Peter Fritzen, Daniele Segneri, Max Pidgeon [4]. Besides the European researchers, there are works of the South Korean scientists Cheol-Hea Koo, Hoon-Hee Lee, Sung-Tae Moon, Sang-Hyuck Han, Gwang-Hyeok Ju [5] from the Korean Institute of Aerospace Design, who also use the SMP standard to create simulation modeling environments.

The analysis of the existing infrastructures of simulation modeling has shown that application of the SMP standard provides great possibilities to build and control models. The openness and fixity of the interaction interfaces of the model and simulation platform allows to create simulation models by their integration between each other. Regulation of the interaction of the models provides the basis for building complex solutions on the basis of the models of different purposes, each containing one of the functional aspects of the object under modeling.

Though development of infrastructures is completed in accordance with the principles set by the standard, in the basis of each of them there lays a unique vision of how the model must be realized, of the ways of its creation and of the internal logics of simulation modeling. The standard regulates the upper level of the modeled object - metamodel. For its description in infrastructures the designers use the SMDL language [6]. The metamodel determines architecture, hierarchy and configuration of connections of different models in one whole. On the basis of the metamodel, the simulation modeling infrastructure determines interaction of different components of the model and the rules of calling simulation procedures. The models used in the metamodel are equipped with the unique mechanisms of their internal realization, methods of control over separate elements of models and transitions between the modeled conditions, not regulated by the SMP2 standard.

The authors are experienced in building simulation models designed to model spacecraft command and measuring systems' onboard equipment function. This experience has 
become the basis for making researches on creating our own infrastructure of simulation modeling. The analysis has shown that in order to work with the existing infrastructures, the user must be qualified in onboard system design and in programming, because creation of simulation models requires usage of high-level programming languages. Such approach makes it difficult to build or make changes in the models. We suggest to use intellectual methods of presenting the logics of the model functioning. With this approach, the model will be described in the terms of the subject area and automatically translated in the program elements working in accordance with the SMP standard. To realize this approach, we have made a formal description of the onboard equipment model and a unified presentation of this model in the SMP2 standard.

\section{UNIFIED PRESENTATION OF ONBOARD EQUIPMENT MODEL IN SMP 2 STANDARD}

\section{A. Model Structure}

In order to create a formal model of onboard equipment we studied its functional features during normal operation as well as under external command-and-software control. We studied different aspects of the modelled systems that would allow to provide support to construction and testing of the onboard equipment.

The model structure $M=<S, R>$ includes structuralparametric description $S=<D, G, I, C, P, T>$ and functional description $\mathrm{R}$, containing the rules of the model completion.

In the given description $D$ is a number of data structures; $G$ is the set of subsystems (elements) of the model; $I$ is the number of communication interfaces corresponding with the input and output points of the model's elements; $C$ is the number of links between communication interfaces determining directions of the data flows; $P$ - variables and $T-$ timers.

The functional description of the model is based on its structural-parametric description. Function description $R=\{A \rightarrow B\}$ determines the algorithms of model's subsystems' behavior and is a knowledge base consisting of a set of rules (condition-action rules) [7]. Each rule is set by a symbol construction $A \rightarrow B$, where $A$ is a condition and $B$ is an action. $A$ determines the condition of the model's elements in which a given rule can be applied. The right part of the rule $B$ sets the actions determining the proper step of task solution or the way of changing the model's condition. Conditions and actions are the expression on variables, set in a structural-parametric description of the model, or the functions performing changes in the model's condition.

This model structure is designed in accordance with the requirements of the subject area of spacecraft onboard equipment construction. It can be used for simulation modeling of the processes of onboard systems' function. In order to expand the area of simulation models' usage and their integration into one infrastructure based on the SMP2 standard, it is necessary to add the model with the unification components allowing to describe the control elements, links and possible effects on the model.

\section{B. Model Unification Components}

A unified presentation of the model will make it possible to realize the modeling infrastructure subsystems such as graphics builder, planner, event recording module, data visualizer and model action simulator, as well as to set the parameters of simulation control and modeling of external impacts and emergency situations.

The authors have studied the examples of infrastructures where different approaches to model building are used. In the SWARMSIM [8] project the model is the only source of production for the whole system. Graphical presentation of the model is realized with the help of the UML language. Software components allow to make a controllable integration of the parts of business logics with the part of model generation. All further project changes are completed at the graphical presentation of the model.

We plan to use an information-and-graphic presentation of the model demonstrating the real structure of onboard equipment, its composition and features, both for the stage of the model building and during the process of simulation modeling. During simulation, the model visualizes the current processes, command transmission and execution, change of parameters and data packages. In order to imbed such model in infrastructure and support the SMP2 standard, we have made a unification of the model's elements.

The SMP standard includes five approaches to model building and integration:

- lass-based design,

- interface-based design,

- component-based design,

- event-based design,

- dataflow-based design.

The unified model is an interface-based design, as it must meet the requirements of the onboard equipment engineers and be very close to the modeled object both functionally and structurally. Figure I shows the components of the unified model. The metamodel in SMDL format contains description of separate models, their input points and communication interfaces. Wrapper is an intermediate link that provides calls of the functions declared in the metamodel by transferring control to the dynamic linked library of model. Wrapper is generated automatically on the basis of the built model by means of generation and compilation of code in the $\mathrm{C}++$ language.

Dynamic Linked Library of model realizes the mechanisms providing model's function. It includes the mechanisms of logical inference and of interaction between the model's elements through communication interfaces.

The Dynamic Linked Library of model is tuned in accordance with the configuration containing structural description of the model in the form of blocks and communication interfaces and functional description in the form of a base of rules. These components allow to create, integrate and jointly use the simulation models of different 
purpose.

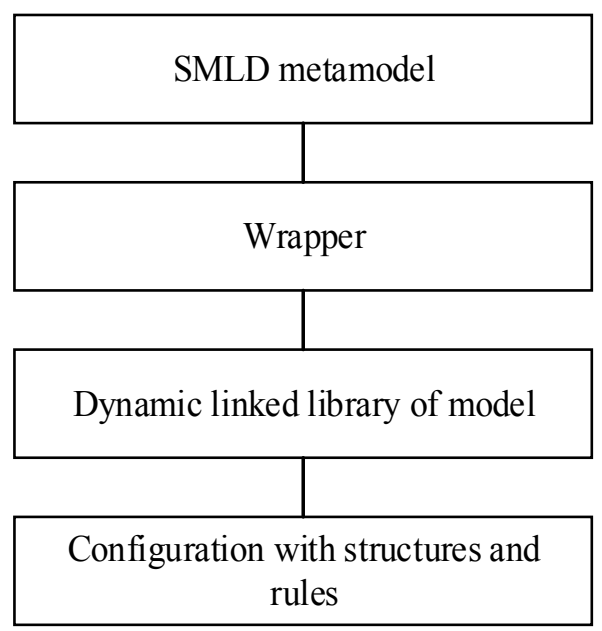

FIGURE I. MODEL UNIFICATION COMPONENTS

The modeling infrastructure will become a base for accumulation and generalization of the test results and knowledge bases consolidating the experience in creation of satellite systems' onboard equipment.

\section{ONBOARD EQUIPMENT MODEL EXAMPLE}

Let's study an example of a spacecraft onboard equipment model construction. At the stage of onboard systems' functions and properties design, and in order to analyze their work in the process of testing, it is necessary to model the actions of the systems executing commands of the Ground control complex. For this purpose, we need to build a model simulating command and software control of onboard equipment.

The graphical modeling tools developed by the authors are used to make configuration and tuning of the model: setting of data transmission commutation interfaces, links and parameters of the modeled equipment [9].

Figure II demonstrates a part of the onboard equipment model designed to simulate external command and software control. The following symbols are used: OCS CU - onboard control complex, CCU - command and measuring system's interface module, ODGS - onboard remote signaling equipment, GCC - Ground control complex, TRANS Transmitter, RECIV - Receiver. The connections between the graphical elements are the directions of information exchange during transmission of data packages. The model has a number of parameters and characteristics that can be changed, thus setting different configurations of spacecraft command-andmeasuring system's onboard equipment.

The logics of the model's function is described in the knowledge base allowing to record different characteristics of its function. The knowledge base contains the rules of reception, transmission and execution of the commands transferred from the Ground control complex to the onboard systems. For example:

\section{Rule 1:}

If interface «Input» has received a command and the type of command $=$ «Command OCS CU», then produce the interface command «To OCS CU»

\section{Rule 2:}

If interface «Input» has received a command and the type of command $=$ «Command CCU» and the command $=« \mathrm{On}$ TRANS» then produce the interface impulse «TRANS Control )

The possibility to set the logics of function in a form of rules makes it easy for an engineer qualified in the work of a spacecraft's onboard equipment, to build and then modify a simulation model without special knowledge in programming. The rules are clear and simple, allowing the engineer to understand the structure of his own models as well as of the models created by other specialists, and also to see the modeled system in whole.

\section{ACKNOWLEDGMENT}

The reported study was funded by RFBR and Government of Krasnoyarsk Territory according to the research project № 16-41-242042.

\section{REFERENCES}

[1] Simulation modelling platform. ECSS E-40-07 // ESA Requirements and Standards Division ESTEC, The Netherlands, 2011, p. 49.

[2] C. Cazenave and W. Arrouy, "Implementing SMP2 Standard within SimTG Simulation Infrastructure," SESP (ESA) : Simulation and EGSE for Space Programmes, 2012.

[3] J. Eggleston, H. Boyer, D. van der Zee, A. Pidgeon, N. de Nisio, F. Burro, and N. Lindman, "Simsat 3.0: Esoc's New Simulation Infrastructure," 6th International Symposium on Reducing the Costs of Spacecraft Ground Systems and Operations (RCSGSO)

[4] Peter Fritzen, Daniele Segneri and Max Pignède, "SWARMSIM - The first fully SMP2 based Simulator for ESOC," 11th Int. WS on Simulation \& EGSE facilities for Space Programmes SESP, 2010.

[5] Cheol-Hea Koo, Hoon-Hee Lee, Sung-Tae Moon, Sang-Hyuck Han and Gwang-Hyeok Ju, "Development of simulation infrastructure compatible with ESA SMP for validation of flight software and verification of mission operation" SESP (ESA) : Simulation and EGSE for Space Programmes, 2012.

[6] Simulation modelling platform - Volume 2a: Metamodel. ECSS E-40-07 // ESA Requirements and Standards Division ESTEC, The Netherlands, 2011, p. 169.

[7] Russell and J. Stuart, Artificial intelligence: a modern approach, Prentice-Hall, Inc. A Simon \& Schuster Company Englewood Cliffs: New Jersey, p. $932,1995$.

[8] M. Pignède, J. Morales, P.Fritzen and J.Lewis, "Swarm Constellation Simulator," SpaceOps 2010 Conference Delivering on the Dream Hosted by NASA Marshall Space Flight Center and Organized by AIAA, 2010, p. 2323.

[9] L. Nozhenkova, O. Isaeva and E.Gruzenko, "Computer Simulation of Spacecraft Onboard Equipment," Proceedings series Advances in Computer Science Research, vol.18, 2015, pp. 943-945. 


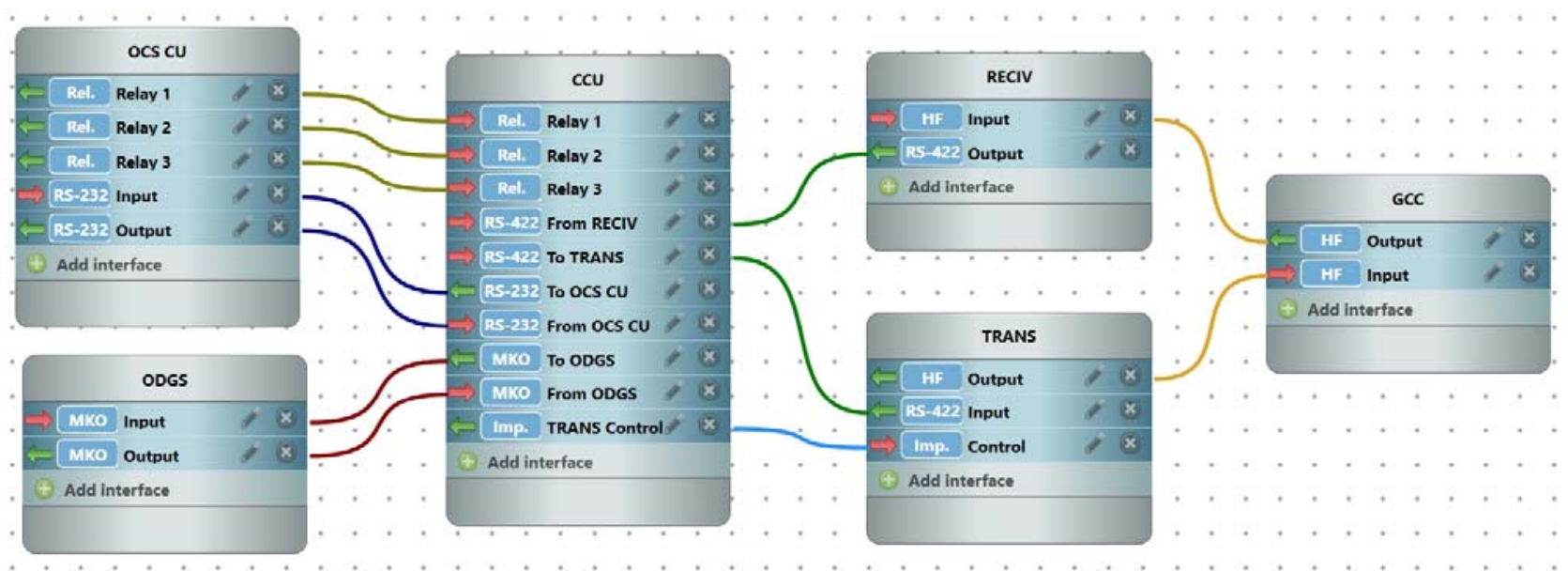

FIGURE II. A PART OF ONBOARD EQUIPMENT MODEL FOR COMMAND-AND-SOFTWARE CONTROL SIMULATION 